\title{
The Solution Set Characterization and Error Bound for the Extended Mixed Linear Complementarity Problem
}

\author{
Hongchun Sun ${ }^{1}$ and Yiju Wang ${ }^{2}$ \\ ${ }^{1}$ School of Sciences, Linyi University, Linyi, Shandong 276005, China \\ ${ }^{2}$ School of Management Science, Qufu Normal University, Rizhao, Shandong 276800, China
}

Correspondence should be addressed to Yiju Wang, wyiju@hotmail.com

Received 19 September 2012; Accepted 8 December 2012

Academic Editor: Jian-Wen Peng

Copyright (C) 2012 H. Sun and Y. Wang. This is an open access article distributed under the Creative Commons Attribution License, which permits unrestricted use, distribution, and reproduction in any medium, provided the original work is properly cited.

For the extended mixed linear complementarity problem (EML CP), we first present the characterization of the solution set for the EMLCP. Based on this, its global error bound is also established under milder conditions. The results obtained in this paper can be taken as an extension for the classical linear complementarity problems.

\section{Introduction}

We consider that the extended mixed linear complementarity problem, abbreviated as EMLCP, is to find vector $\left(x^{*} ; y^{*}\right) \in R^{2 n}$ such that

$$
\begin{gathered}
F\left(x^{*}\right) \geq 0, \quad G\left(x^{*}, y^{*}\right) \geq 0, \quad F\left(x^{*}\right)^{\top} G\left(x^{*}, y^{*}\right)=0, \\
A x^{*}+B y^{*}+b \geq 0, \quad C x^{*}+D y^{*}+d=0,
\end{gathered}
$$

where $F(x)=M x+p, G(x)=N x+Q y+q, M, N, Q \in R^{m \times n}, p, q \in R^{m}, A, B \in R^{s \times n}, C, D \in$ $R^{t \times n}, b \in R^{s}, d \in R^{t}$. We assume that the solution set of the EMLCP is nonempty throughout this paper.

The EMLCP is a direct generalization of the classical linear complementarity problem and a special case of the generalized nonlinear complementarity problem which was discussed in the literature $([1,2])$. The extended complementarity problem plays a significant role in economics, engineering, and operation research, and so forth [3]. For example, 
the balance of supply and demand is central to all economic systems; mathematically, this fundamental equation in economics is often described by a complementarity relation between two sets of decision variables. Furthermore, the classical Walrasian law of competitive equilibria of exchange economies can be formulated as a generalized nonlinear complementarity problem in the price and excess demand variables [4].

Up to now, the issues of the solution set characterization and numerical methods for the classical linear complementarity problem or the classical nonlinear complementarity problem were fully discussed in the literature (e.g., [5-8]). On the other hand, the global error bound is also an important tool in the theoretical analysis and numerical treatment for variational inequalities, nonlinear complementarity problems, and other related optimization problems [9]. The error bound estimation for the classical linear complementarity problems (LCP) was fully analyzed (e.g., [7-12]).

Obviously, the EMLCP is an extension of the LCP, and this motivates us to extend the solution set characterization and error bound estimation results of the LCP to the EMLCP. To this end, we first detect the solution set characterization of the EMLCP under milder conditions in Section 2. Based on these, we establish the global error bound estimation for the EMLCP in Section 3. These constitute what can be taken as an extension of those for linear complementarity problems.

We end this section with some notations used in this paper. Vectors considered in this paper are all taken in Euclidean space equipped with the standard inner product. The Euclidean norm of vector in the space is denoted by $\|\cdot\|$. We use $R_{+}^{n}$ to denote the nonnegative orthant in $R^{n}$ and use $x_{+}$and $x_{-}$to denote the vectors composed by elements $\left(x_{+}\right)_{i}:=\max \left\{x_{i}, 0\right\}$ and $\left(x_{-}\right)_{i}:=\max \left\{-x_{i}, 0\right\}, 1 \leq i \leq n$, respectively. For simplicity, we use $(x ; y)$ for column vector $\left(x^{\top}, y^{\top}\right)^{\top}$. We also use $x \geq 0$ to denote a nonnegative vector $x \in R^{n}$ if there is no confusion.

\section{The Solution Set Characterization for EMLCP}

In this section, we will characterize the solution set of the EMLCP. First, we can give the needed assumptions for our analysis.

Assumption 2.1. For the matrices $M, N, Q$ involved in the EMLCP, we assume that the matrix $\left(\begin{array}{cc}M^{\top} N+N^{\top} M & M^{\top} Q \\ Q^{\top} M & 0\end{array}\right)$ is positive semidefinite.

Theorem 2.2. Suppose that Assumption 2.1 holds; the following conclusions hold.

(i) If $\left(x_{0} ; y_{0}\right)$ is a solution of the EMLCP, then

$$
\begin{gathered}
X^{*}=\left\{(x ; y) \in X \mid\left\{\left(M, 0_{m \times n}\right)^{\top}(N, Q)+(N, Q)^{\top}\left(M, 0_{m \times n}\right)\right\}\left\{(x ; y)-\left(x_{0} ; y_{0}\right)\right\}=0,\right. \\
\left.\left\{\left(M, 0_{m \times n}\right)^{\top} q+(N, Q)^{\top} p\right\}^{\top}\left\{(x ; y)-\left(x_{0} ; y_{0}\right)\right\}=0\right\},
\end{gathered}
$$

where $X=\left\{(x ; y) \in R^{2 n} \mid M x+p \geq 0, N x+Q y+q \geq 0, A x+B y+b \geq 0, C x+D y+d=0\right\}$, and $X^{*}$ denotes the solution set of EMLCP. 
Journal of Applied Mathematics

(ii) If $\left(x_{1} ; y_{1}\right)$ and $\left(x_{2} ; y_{2}\right)$ are two solutions of the EMLCP, then

$$
\left(M x_{1}+p\right)^{\top}\left(N x_{2}+Q y_{2}+q\right)=\left(M x_{2}+p\right)^{\top}\left(N x_{1}+Q y_{1}+q\right)=0
$$

(iii) The solution set of EMLCP is convex.

Proof. (i) Set

$$
\begin{gathered}
W=\left\{(x ; y) \in X \mid\left\{(M, 0)^{\top}(N, Q)+(N, Q)^{\top}(M, 0)\right\}\left\{(x ; y)-\left(x_{0} ; y_{0}\right)\right\}=0,\right. \\
\left.\left\{(M, 0)^{\top} q+(N, Q)^{\top} p\right\}^{\top}\left\{(x ; y)-\left(x_{0} ; y_{0}\right)\right\}=0\right\} .
\end{gathered}
$$

For any $(\tilde{x} ; \tilde{y}) \in X^{*}$, since $\left(x_{0} ; y_{0}\right) \in X$, we have

$$
\begin{aligned}
& \left(\left(x_{0} ; y_{0}\right)-(\tilde{x} ; \tilde{y})\right)^{\top}\left((M, 0)^{\top}(N, Q)(\tilde{x} ; \tilde{y})+(M, 0)^{\top} q\right) \\
& \quad=\left[\left(M x_{0}+p\right)-(M \tilde{x}+p)\right]^{\top}(N \tilde{x}+Q \tilde{y}+q) \\
& \quad=\left[\left(M x_{0}+p\right)\right]^{\top}(N \tilde{x}+Q \tilde{y}+q)-(M \tilde{x}+p)^{\top}(N \tilde{x}+Q \tilde{y}+q) \\
& \quad=\left[\left(M x_{0}+p\right)\right]^{\top}(N \tilde{x}+Q \tilde{y}+q) \geq 0 .
\end{aligned}
$$

Since $(\tilde{x} ; \tilde{y}) \in X,\left(x_{0} ; y_{0}\right) \in X^{*}$, using the similar arguments to that in $(2.4)$, we have

$$
\left((\tilde{x} ; \tilde{y})-\left(x_{0} ; y_{0}\right)\right)^{\top}\left((M, 0)^{\top}(N, Q)\left(x_{0} ; y_{0}\right)+(M, 0)^{\top} q\right) \geq 0
$$

Combining (2.4) with (2.5), one has

$$
\left((\tilde{x} ; \tilde{y})-\left(x_{0} ; y_{0}\right)\right)^{\top}(M, 0)^{\top}(N, Q)\left((\tilde{x} ; \tilde{y})-\left(x_{0} ; y_{0}\right)\right) \leq 0
$$

By (2.6), we have

$$
\left((\tilde{x} ; \tilde{y})-\left(x_{0} ; y_{0}\right)\right)^{\top}\left((M, 0)^{\top}(N, Q)+(N, Q)^{\top}(M, 0)\right)\left((\tilde{x} ; \tilde{y})-\left(x_{0} ; y_{0}\right)\right) \leq 0
$$

By Assumption 2.1, one has

$$
\begin{aligned}
& \left((\tilde{x} ; \tilde{y})-\left(x_{0} ; y_{0}\right)\right)^{\top}\left((M, 0)^{\top}(N, Q)+(N, Q)^{\top}(M, 0)\right)\left((\tilde{x} ; \tilde{y})-\left(x_{0} ; y_{0}\right)\right) \\
& \quad=\left((\tilde{x} ; \tilde{y})-\left(x_{0} ; y_{0}\right)\right)^{\top}\left(\begin{array}{cc}
M^{\top} N+N^{\top} M & M^{\top} Q \\
Q^{\top} M & 0
\end{array}\right)\left((\tilde{x} ; \tilde{y})-\left(x_{0} ; y_{0}\right)\right) \geq 0 .
\end{aligned}
$$


Combining (2.7) with (2.8), we have

$$
\left((\tilde{x} ; \tilde{y})-\left(x_{0} ; y_{0}\right)\right)^{\top}\left((M, 0)^{\top}(N, Q)+(N, Q)^{\top}(M, 0)\right)\left((\tilde{x} ; \tilde{y})-\left(x_{0} ; y_{0}\right)\right)=0
$$

That is,

$$
\left((M, 0)^{\top}(N, Q)+(N, Q)^{\top}(M, 0)\right)\left((\tilde{x} ; \tilde{y})-\left(x_{0} ; y_{0}\right)\right)=0
$$

Using $\left(x_{0} ; y_{0}\right) \in X,(\tilde{x} ; \tilde{y}) \in X^{*}$ again, we have

$$
\begin{aligned}
\left(\left(x_{0} ; y_{0}\right)-(\tilde{x} ; \tilde{y})\right)^{\top}\left((N, Q)^{\top}(M, 0)(\tilde{x} ; \tilde{y})+(N, Q)^{\top} p\right) \\
\quad=\left[\left(N x_{0}+Q y_{0}+q\right)-(N \tilde{x}+Q \tilde{y}+q)\right]^{\top}(M \tilde{x}+p) \\
\quad=\left(N x_{0}+Q y_{0}+q\right)^{\top}(M \tilde{x}+p)-(N \tilde{x}+Q \tilde{y}+q)^{\top}(M \tilde{x}+p) \\
\quad=\left(N x_{0}+Q y_{0}+q\right)^{\top}(M \tilde{x}+p) \geq 0 .
\end{aligned}
$$

Using $(\tilde{x} ; \tilde{y}) \in X,\left(x_{0} ; y_{0}\right) \in X^{*}$ again, using the similar arguments to that in $(2.11)$, we have

$$
\left((\tilde{x} ; \tilde{y})-\left(x_{0} ; y_{0}\right)\right)^{\top}\left((N, Q)^{\top}(M, 0)\left(x_{0} ; y_{0}\right)+(N, Q)^{\top} p\right) \geq 0
$$

From (2.9), (2.4), and (2.11), one has

$$
\begin{gathered}
\left(\left(x_{0} ; y_{0}\right)-(\tilde{x} ; \tilde{y})\right)^{\top}\left\{\left((M, 0)^{\top}(N, Q)+(N, Q)^{\top}(M, 0)\right)\left(x_{0} ; y_{0}\right)+(M, 0)^{\top} q+(N, Q)^{\top} p\right\} \\
=\left(\left(x_{0} ; y_{0}\right)-(\tilde{x} ; \tilde{y})\right)^{\top}\left((M, 0)^{\top}(N, Q)+(N, Q)^{\top}(M, 0)\right)\left(\left(x_{0} ; y_{0}\right)-(\tilde{x} ; \tilde{y})\right) \\
+\left(\left(x_{0} ; y_{0}\right)-(\tilde{x} ; \tilde{y})\right)^{\top}\left\{\left((M, 0)^{\top}(N, Q)+(N, Q)^{\top}(M, 0)\right)(\tilde{x} ; \tilde{y})\right. \\
\left.+(M, 0)^{\top} q+(N, Q)^{\top} p\right\} \\
=\left(\left(x_{0} ; y_{0}\right)-(\tilde{x} ; \tilde{y})\right)^{\top}\left\{(M, 0)^{\top}(N, Q)(\tilde{x} ; \tilde{y})+(M, 0)^{\top} q\right\} \\
+\left(\left(x_{0} ; y_{0}\right)-(\tilde{x} ; \tilde{y})\right)^{\top}\left\{(N, Q)^{\top}(M, 0)(\tilde{x} ; \tilde{y})+(N, Q)^{\top} p\right\} \geq 0 .
\end{gathered}
$$

Combining (2.5) with (2.12) yields

$$
\left((\tilde{x} ; \tilde{y})-\left(x_{0} ; y_{0}\right)\right)^{\top}\left(\left((M, 0)^{\top}(N, Q)+(N, Q)^{\top}(M, 0)\right)\left(x_{0} ; y_{0}\right)+(M, 0)^{\top} q+(N, Q)^{\top} p\right) \geq 0
$$


Journal of Applied Mathematics

Combining this with (2.13) yields

$$
\left((\tilde{x} ; \tilde{y})-\left(x_{0} ; y_{0}\right)\right)^{\top}\left(\left((M, 0)^{\top}(N, Q)+(N, Q)^{\top}(M, 0)\right)\left(x_{0} ; y_{0}\right)+(M, 0)^{\top} q+(N, Q)^{\top} p\right)=0
$$

From (2.10) and (2.15), one has

$$
\left((M, 0)^{\top} q+(N, Q)^{\top} p\right)^{\top}\left((\tilde{x} ; \tilde{y})-\left(x_{0} ; y_{0}\right)\right)=0 .
$$

By (2.10) and (2.16), we obtain that $(\tilde{x} ; \tilde{y}) \in W$ follows.

On the other hand, for any $(\hat{x} ; \widehat{y}) \in W$, then $(\widehat{x} ; \widehat{y}) \in X$, and

$$
\begin{gathered}
\left((M, 0)^{\top}(N, Q)+(N, Q)^{\top}(M, 0)\right)\left((\widehat{x} ; \widehat{y})-\left(x_{0} ; y_{0}\right)\right)=0 \\
\left((M, 0)^{\top} q+(N, Q)^{\top} p\right)\left((\widehat{x} ; \widehat{y})-\left(x_{0} ; y_{0}\right)\right)=0
\end{gathered}
$$

and one has

$$
\begin{aligned}
0= & \left((\widehat{x} ; \widehat{y})-\left(x_{0} ; y_{0}\right)\right)^{\top}\left[\left((M, 0)^{\top}(N, Q)+(N, Q)^{\top}(M, 0)\right)\left(x_{0} ; y_{0}\right)\right. \\
& \left.+(M, 0)^{\top} q+(N, Q)^{\top} p\right] \\
= & \left((\widehat{x} ; \hat{y})-\left(x_{0} ; y_{0}\right)\right)^{\top}\left((M, 0)^{\top}(N, Q)\left(x_{0} ; y_{0}\right)+(M, 0)^{\top} q\right) \\
& +\left((\widehat{x} ; \widehat{y})-\left(x_{0} ; y_{0}\right)\right)^{\top}\left((N, Q)^{\top}(M, 0)\left(x_{0} ; y_{0}\right)+(N, Q)^{\top} p\right) \\
= & {\left[(M \widehat{x}+p)-\left(M x_{0}+p\right)\right]^{\top}\left(N x_{0}+Q y_{0}+q\right) } \\
& \left.+\left[(N \hat{x}+Q \widehat{y}+q)-\left(N x_{0}+Q y_{0}\right)+q\right)\right]^{\top}\left(M x_{0}+p\right) \\
= & (M \widehat{x}+p)^{\top}\left(N x_{0}+Q y_{0}+q\right)+(N \hat{x}+Q \widehat{y}+q)^{\top}\left(M x_{0}+p\right) .
\end{aligned}
$$

Using (2.18), one has

$$
\begin{aligned}
0= & \left((\widehat{x} ; \widehat{y})-\left(x_{0} ; y_{0}\right)\right)^{\top}\left((M, 0)^{\top}(N, Q)+(N, Q)^{\top}(M, 0)\right)\left((\widehat{x} ; \widehat{y})-\left(x_{0} ; y_{0}\right)\right) \\
= & 2\left((\widehat{x} ; \widehat{y})-\left(x_{0} ; y_{0}\right)\right)^{\top}(M, 0)^{\top}(N, Q)\left((\widehat{x} ; \widehat{y})-\left(x_{0} ; y_{0}\right)\right) \\
= & 2\left[(M \widehat{x}+p)-\left(M x_{0}+p\right)\right]^{\top}\left[(N \widehat{x}+Q \widehat{y}+q)-\left(N x_{0}+Q y_{0}+q\right)\right] \\
= & 2\left[(M \widehat{x}+p)^{\top}(N \hat{x}+Q \widehat{y}+q)-(M \widehat{x}+p)^{\top}\left(N x_{0}+Q y_{0}+q\right)\right. \\
& \left.\quad-\left(M x_{0}+p\right)^{\top}(N \hat{x}+Q \widehat{y}+q)+\left(M x_{0}+p\right)^{\top}\left(N x_{0}+Q y_{0}+q\right)\right] \\
= & 2(M \widehat{x}+p)^{\top}(N \hat{x}+Q \widehat{y}+q) .
\end{aligned}
$$

Thus, we have that $(\widehat{x} ; \widehat{y}) \in X^{*}$. 

have

(ii) Since $\left(x_{1} ; y_{1}\right)$ and $\left(x_{2} ; y_{2}\right)$ are two solutions of the EMLCP, by Theorem 2.2 (i), we

$$
\begin{aligned}
\left((M, 0)^{\top}(N, Q)+(N, Q)^{\top}(M, 0)\right)\left(\left(x_{1} ; y_{1}\right)-\left(x_{2} ; y_{2}\right)\right) \\
=\left((M, 0)^{\top}(N, Q)+(N, Q)^{\top}(M, 0)\right)\left(\left(x_{1} ; y_{1}\right)-\left(x_{0} ; y_{0}\right)\right) \\
\quad-\left((M, 0)^{\top}(N, Q)+(N, Q)^{\top}(M, 0)\right)\left(\left(x_{2} ; y_{2}\right)-\left(x_{0} ; y_{0}\right)\right)=0 .
\end{aligned}
$$

Combining this with $\left(M x_{1}+p\right)^{\top}\left(N x_{1}+Q y_{1}+q\right)=\left(M x_{2}+p\right)^{\top}\left(N x_{2}+Q y_{2}+q\right)=0$, one has

$$
\begin{aligned}
0 & =\left(\left(x_{1} ; y_{1}\right)-\left(x_{2} ; y_{2}\right)\right)^{\top}\left((M, 0)^{\top}(N, Q)+(N, Q)^{\top}(M, 0)\right)\left(\left(x_{1} ; y_{1}\right)-\left(x_{2} ; y_{2}\right)\right) \\
& =2\left(\left(x_{1} ; y_{1}\right)-\left(x_{2} ; y_{2}\right)\right)^{\top}(M, 0)^{\top}(N, Q)\left(\left(x_{1} ; y_{1}\right)-\left(x_{2} ; y_{2}\right)\right) \\
& =2\left[\left(M x_{1}+p\right)-\left(M x_{2}+p\right)\right]^{\top}\left[\left(N x_{1}+Q y_{1}+q\right)-\left(N x_{2}+Q y_{2}+q\right)\right] \\
& =-2\left[\left(M x_{1}+p\right)^{\top}\left(N x_{2}+Q y_{2}+q\right)+\left(M x_{2}+p\right)^{\top}\left(N x_{1}+Q y_{1}+q\right)\right] .
\end{aligned}
$$

On the other hand, from $M x_{i}+p \geq 0, N x_{i}+Q y_{i}+q \geq 0, i=1,2$, we can deduce

$$
\left(M x_{1}+p\right)^{\top}\left(N x_{2}+Q y_{2}+q\right) \geq 0, \quad\left(M x_{2}+p\right)^{\top}\left(N x_{1}+Q y_{1}+q\right) \geq 0
$$

From (2.21) and (2.22), thus, we have that Theorem 2.2 (ii) holds.

(iii) If solution set of the EMLCP is single point set, then it is obviously convex. In this following, we suppose that $\left(x^{1} ; y^{1}\right)$ and $\left(x^{2} ; y^{2}\right)$ are two solutions of the EMLCP. By Theorem 2.2 (i), we have

$$
\begin{gathered}
\left((M, 0)^{\top}(N, Q)+(N, Q)^{\top}(M, 0)\right)\left(\left(x^{1} ; y^{1}\right)-\left(x_{0} ; y_{0}\right)\right)=0, \\
\left((M, 0)^{\top}(N, Q)+(N, Q)^{\top}(M, 0)\right)\left(\left(x^{2} ; y^{2}\right)-\left(x_{0} ; y_{0}\right)\right)=0, \\
\left((M, 0)^{\top} q+(N, Q)^{\top} p\right)^{\top}\left(\left(x^{1} ; y^{1}\right)-\left(x_{0} ; y_{0}\right)\right)=0 \\
\left((M, 0)^{\top} q+(N, Q)^{\top} p\right)^{\top}\left(\left(x^{2} ; y^{2}\right)-\left(x_{0} ; y_{0}\right)\right)=0 .
\end{gathered}
$$

For the vector $(x ; y)=\tau\left(x^{1} ; y^{1}\right)+(1-\tau)\left(x^{2} ; y^{2}\right)$, for all $\tau \in[0,1]$, by $(2.23)$, we have

$$
\begin{aligned}
& \left((M, 0)^{\top}(N, Q)+(N, Q)^{\top}(M, 0)\right)\left((x ; y)-\left(x_{0} ; y_{0}\right)\right) \\
& =\left((M, 0)^{\top}(N, Q)+(N, Q)^{\top}(M, 0)\right)\left(\tau\left(x^{1} ; y^{1}\right)-\tau\left(x_{0} ; y_{0}\right)\right) \\
& \quad+\left((M, 0)^{\top}(N, Q)+(N, Q)^{\top}(M, 0)\right)\left((1-\tau)\left(x^{2} ; y^{2}\right)-(1-\tau)\left(x_{0} ; y_{0}\right)\right)=0
\end{aligned}
$$


Journal of Applied Mathematics

Using the similar arguments to that in (2.24), we can also obtain

$$
\left((M, 0)^{\top} q+(N, Q)^{\top} p\right)^{\top}\left((x ; y)-\left(x_{0} ; y_{0}\right)\right)=0 .
$$

Combining (2.24) and (2.25) with the conclusion of Theorem 2.2(i), we obtain the desired result.

Corollary 2.3. Suppose that Assumption 2.1 holds. Then, the solution set for EMLCP has the following characterization:

$$
\begin{gathered}
X^{*}=\left\{(x ; y) \in X \mid\left((M, 0)^{\top}(N, Q)+(N, Q)^{\top}(M, 0)\right)\left((x ; y)-\left(x_{0} ; y_{0}\right)\right)=0\right. \\
\left((x ; y)-\left(x_{0} ; y_{0}\right)\right)^{\top}\left[\left((M, 0)^{\top}(N, Q)+(N, Q)^{\top}(M, 0)\right)\left(x_{0} ; y_{0}\right)\right. \\
\left.\left.+(M, 0)^{\top} q+(N, Q)^{\top} p\right] \leq 0 .\right\} .
\end{gathered}
$$

Proof. Set

$$
\begin{gathered}
\widetilde{W}=\left\{(x ; y) \in X \mid\left((M, 0)^{\top}(N, Q)+(N, Q)^{\top}(M, 0)\right)\left((x ; y)-\left(x_{0} ; y_{0}\right)\right)=0,\right. \\
\left((x ; y)-\left(x_{0} ; y_{0}\right)\right)^{\top}\left[\left((M, 0)^{\top}(N, Q)+(N, Q)^{\top}(M, 0)\right)\left(x_{0} ; y_{0}\right)\right. \\
\left.\left.+(M, 0)^{\top} q+(N, Q)^{\top} p\right] \leq 0\right\} .
\end{gathered}
$$

For any $(\widehat{x} ; \widehat{y}) \in \widetilde{W}$, then $(\widehat{x} ; \widehat{y}) \in X$, combining this with $\left(x_{0} ; y_{0}\right) \in X^{*}$. Using the similar arguments to that in (2.5) and (2.12), we have

$$
\left((\widehat{x} ; \widehat{y})-\left(x_{0} ; y_{0}\right)\right)^{\top}\left[\left((M, 0)^{\top}(N, Q)+(N, Q)^{\top}(M, 0)\right)\left(x_{0} ; y_{0}\right)+(M, 0)^{\top} q+(N, Q)^{\top} p\right] \geq 0
$$

Combining this with $(\widehat{x} ; \widehat{y}) \in \widetilde{W}$, one has

$$
\left((\widehat{x} ; \widehat{y})-\left(x_{0} ; y_{0}\right)\right)^{\top}\left[\left((M, 0)^{\top}(N, Q)+(N, Q)^{\top}(M, 0)\right)\left(x_{0} ; y_{0}\right)+(M, 0)^{\top} q+(N, Q)^{\top} p\right]=0 .
$$

From $\left((M, 0)^{\top}(N, Q)+(N, Q)^{\top}(M, 0)\right)\left((\widehat{x} ; \widehat{y})-\left(x_{0} ; y_{0}\right)\right)=0$, we have

$$
\left((M, 0)^{\top} q+(N, Q)^{\top} p\right)^{\top}\left((\widehat{x} ; \widehat{y})-\left(x_{0} ; y_{0}\right)\right)=0
$$

Thus, by Theorem $2.2(\mathrm{i})$, one has $(\widehat{x} ; \widehat{y}) \in X^{*}$. 
On the other hand, for any $(\hat{x} ; \hat{y}) \in X^{*}$, by Theorem 2.2 (i), we have $(\hat{x} ; \hat{y}) \in X$, $\left((M, 0)^{\top}(N, Q)+(N, Q)^{\top}(M, 0)\right)\left((\widehat{x} ; \widehat{y})-\left(x_{0} ; y_{0}\right)\right)=0$, and $\left((M, 0)^{\top} q+(N, Q)^{\top} p\right)^{\top}((\hat{x} ; \widehat{y})-$ $\left.\left(x_{0} ; y_{0}\right)\right)=0$, that is,

$$
\left((\widehat{x} ; \widehat{y})-\left(x_{0} ; y_{0}\right)\right)^{\top}\left[\left((M, 0)^{\top}(N, Q)+(N, Q)^{\top}(M, 0)\right)\left(x_{0} ; y_{0}\right)+(M, 0)^{\top} q+(N, Q)^{\top} p\right]=0
$$

Thus, $(\widehat{x} ; \widehat{y}) \in \widetilde{W}$.

Using the following definition developed from EMLCP, we can further detect the solution structure of the EMLCP.

Definition 2.4. A solution $(\bar{x} ; \bar{y})$ of the EMLCP is said to be nondegenerate if it satisfies

$$
(M \bar{x}+p)+(N \bar{x}+Q \bar{y}+q)>0 .
$$

Theorem 2.5. Suppose that Assumption 2.1 holds, and the EMLCP has a nondegenerate solution, say $\left(x_{0} ; y_{0}\right)$. Then, the following conclusions hold.

(i) The solution set of EMLCP

$$
\begin{gathered}
X^{*}=\left\{(x ; y) \in X \mid\left((x ; y)-\left(x_{0} ; y_{0}\right)\right)^{\top}\left[\left((M, 0)^{\top}(N, Q)+(N, Q)^{\top}(M, 0)\right)\left(x_{0} ; y_{0}\right)\right.\right. \\
\left.\left.+(M, 0)^{\top} q+(N, Q)^{\top} p\right] \leq 0\right\}
\end{gathered}
$$

(ii) If the matrices $M_{\bar{\alpha}}$ and $Q_{\alpha}$ are the full-column rank, where $\alpha=\left\{i \mid\left(M x_{0}+p\right)_{i}>0, i=\right.$ $1,2, \ldots, m\}, \bar{\alpha}=\{i \mid i=1,2, \ldots, m, i \notin \alpha\}$, then $\left(x_{0} ; y_{0}\right)$ is the unique nondegenerate solution of EMLCP.

Proof. (i) Set

$$
\begin{gathered}
\bar{W}=\left\{(x ; y) \in X \mid\left((x ; y)-\left(x_{0} ; y_{0}\right)\right)^{\top}\left[\left((M, 0)^{\top}(N, Q)+(N, Q)^{\top}(M, 0)\right)\left(x_{0} ; y_{0}\right)\right.\right. \\
\left.\left.+(M, 0)^{\top} q+(N, Q)^{\top} p\right] \leq 0\right\} .
\end{gathered}
$$

From Corollary 2.3, one has $X^{*} \subseteq \bar{W}$. In this following, we will show that $\bar{W} \subseteq X^{*}$. For any $(x ; y) \in \bar{W}$, then $(x ; y) \in X$, combining this with $\left(x_{0} ; y_{0}\right) \in X^{*}$. Using the similar arguments to that in (2.14), we have

$$
\left((x ; y)-\left(x_{0} ; y_{0}\right)\right)^{\top}\left[\left((M, 0)^{\top}(N, Q)+(N, Q)^{\top}(M, 0)\right)\left(x_{0} ; y_{0}\right)+(M, 0)^{\top} q+(N, Q)^{\top} p\right] \geq 0 .
$$


Combining this with $(x ; y) \in \bar{W}$, one has

$$
\begin{aligned}
0= & \left((x ; y)-\left(x_{0} ; y_{0}\right)\right)^{\top}\left[\left((M, 0)^{\top}(N, Q)+(N, Q)^{\top}(M, 0)\right)\left(x_{0} ; y_{0}\right)+(M, 0)^{\top} q+(N, Q)^{\top} p\right] \\
= & \left((x ; y)-\left(x_{0} ; y_{0}\right)\right)^{\top}\left[(M, 0)^{\top}(N, Q)\left(x_{0} ; y_{0}\right)+(M, 0)^{\top} q\right] \\
& +\left((x ; y)-\left(x_{0} ; y_{0}\right)\right)^{\top}\left[(N, Q)^{\top}(M, 0)\left(x_{0} ; y_{0}\right)+(N, Q)^{\top} p\right] \\
= & {\left[(M x+p)-\left(M x_{0}+p\right)\right]^{\top}\left(N x_{0}+Q y_{0}+q\right) } \\
& +\left[(N x+Q y+q)-\left(N x_{0}+Q y_{0}+q\right)\right]^{\top}\left(M x_{0}+p\right) \\
= & (M x+p)^{\top}\left(N x_{0}+Q y_{0}+q\right)+(N x+Q y+q)^{\top}\left(M x_{0}+p\right) .
\end{aligned}
$$

Combining $M x+p \geq 0, N x+Q y+q \geq 0$ with (2.36), one has

$$
(M x+p)^{\top}\left(N x_{0}+Q y_{0}+q\right)=\left(M x_{0}+p\right)^{\top}(N x+Q y+q)=0
$$

Since $\left(x_{0} ; y_{0}\right)$ is a nondegenerate solution, combining this with $(2.37)$, we have $(M x+$ $p)^{\top}(N x+Q y+q)=0$. That is, $(x ; y) \in X^{*}$.

(ii) Let $(\widehat{x} ; \widehat{y})$ be any nondegenerate solution. Since $\left(x_{0} ; y_{0}\right)$ is a nondegenerate solution, then we have

$$
\begin{aligned}
& \left(M x_{0}+p\right)^{\top}\left(N x_{0}+Q y_{0}+q\right)=0 \\
& \left(M x_{0}+p\right)+\left(N x_{0}+Q y_{0}+q\right)>0
\end{aligned}
$$

Combining (2.38) with (2.39), we have

$$
\left(N x_{0}+Q y_{0}+q\right)_{i}=0, \quad \forall i \in \alpha .
$$

If $i \notin \alpha$, then $\left(N x_{0}+Q y_{0}+q\right)_{i}>0$ by (2.39). By (2.38) again, we can deduce that

$$
\left(M x_{0}+p\right)_{i}=0, \quad \forall i \notin \alpha .
$$

On the other hand, for the $\left(x_{0} ; y_{0}\right)$ and $(\widehat{x} ; \widehat{y})$ which are solutions of EMLCP, and combining Theorem 2.2 (ii), we have $(M \hat{x}+p)^{\top}\left(N x_{0}+Q y_{0}+q\right)=0$. Using $\left(N x_{0}+Q y_{0}+q\right)_{i}>0$, for all $i \notin$ $\alpha$, we can deduce that

$$
(M \widehat{x}+p)_{i}=0, \quad \forall i \notin \alpha .
$$

Combining Theorem 2.2 (ii) again, we also have

$$
\left(\left(M x_{0}+p\right)\right)^{\top}(N \widehat{x}+Q \widehat{y}+q)=0 .
$$


For any $i \in \alpha$, that is, $\left(M x_{0}+p\right)_{i}>0$, and combining (2.43), we obtain

$$
(N \hat{x}+Q \widehat{y}+q)_{i}=0, \quad \forall i \in \alpha
$$

Combining this with the fact that $(M \widehat{x}+p)+(N \hat{x}+Q \widehat{y}+q)>0$, we can deduce that

$$
(M \widehat{x}+p)_{i}>0, \quad \forall i \in \alpha .
$$

From (2.41) and (2.42), we obtain

$$
M_{\bar{\alpha}}\left(\widehat{x}-x_{0}\right)=0 .
$$

Thus, $\widehat{x}=x_{0}$ by the full-column rank assumption on $M_{\bar{\alpha}}$. Using $\widehat{x}=x_{0}$, combining (2.40) with (2.44), we can deduce that

$$
Q_{\alpha} \hat{y}=-N_{\alpha} \widehat{x}-q=-N_{\alpha} x_{0}-q=Q_{\alpha} y_{0} .
$$

That is, $\widehat{y}=y_{0}$ by the full-column rank assumption on $Q_{\alpha}$. Thus, the desired result follows.

The solution set characterization obtained in Theorem 2.2 (i) coincides with that of Lemma 2.1 in [7], and the solution set characterization obtained in Theorem 2.5 (i) coincides with that of Lemma 2.2 in [8] for the linear complementarity problem.

\section{Global Error Bound for the EMLCP}

In this following, we will present a global error bound for the EMLCP based on the results obtained in Corollary 2.3 and Theorem 2.5 (i). Firstly, we can give the needed error bound for a polyhedral cone from [13] and following technical lemmas to reach our claims.

Lemma 3.1. For polyhedral cone $P=\left\{x \in R^{n} \mid D_{1} x=d_{1}, B_{1} x \leq b_{1}\right\}$ with $D_{1} \in R^{l \times n}, B_{1} \in R^{m \times n}$, $d_{1} \in R^{l}$ and $b_{1} \in R^{m}$, there exists a constant $c_{1}>0$ such that

$$
\operatorname{dist}(x, P) \leq c_{1}\left[\left\|D_{1} x-d_{1}\right\|+\left\|\left(B_{1} x-b_{1}\right)_{+}\right\|\right] \quad \forall x \in R^{n} ;
$$

Lemma 3.2. Suppose that $\left(x_{0} ; y_{0}\right)$ is a solution of EMLCP, and let

$$
\omega=\left[\left((M, 0)^{\top}(N, Q)+(N, Q)^{\top}(M, 0)\right)\left(x_{0} ; y_{0}\right)+(M, 0)^{\top} q+(N, Q)^{\top} p\right]
$$

then, there exists a constant $\tau>0$, such that for any $(x ; y) \in R^{2 n}$, one has

$$
\begin{aligned}
& {\left[\omega^{\top}\left((x ; y)-\left(x_{0} ; y_{0}\right)\right)\right]_{-}} \\
& \quad \leq \tau\left(\left\|(M x+p)_{-}\right\|+\left\|(N x+Q y+q)_{-}\right\|+\left\|(A x+B y+b)_{-}\right\|+\|C x+D y+d\|\right) .
\end{aligned}
$$


Proof. Similar to the proof of (2.14), we can obtain

$$
\omega^{\top}\left((x ; y)-\left(x_{0} ; y_{0}\right)\right) \geq 0, \quad \forall(x ; y) \in X
$$

We consider the following linear programming problems

$$
\begin{array}{ll}
\min & \omega^{\top}(x ; y) \\
\text { s.t. } & M x+p \geq 0 \\
& N x+Q y+q \geq 0 \\
& A x+B y+b \geq 0, \\
& C x+D y+d=0
\end{array}
$$

From the assumption, we know that $\left(x_{0}, y_{0}\right)$ is an optimal point of the linear programming problem. Thus, there exist optimal Lagrange multipliers $\lambda_{1}, \lambda_{2} \in R_{+}^{m}, \lambda_{3} \in R_{+}^{s}$, and $\lambda_{4} \in R^{t}$ such that

$$
\begin{gathered}
\omega=(M, 0)^{\top} \lambda_{1}+(N, Q)^{\top} \lambda_{2}+(A, B)^{\top} \lambda_{3}+(C, D)^{\top} \lambda_{4}, \\
M x_{0}+p \geq 0, \quad N x_{0}+Q y_{0}+q \geq 0, \\
A x_{0}+B y_{0}+b \geq 0, \quad C x_{0}+D y_{0}+d=0 \\
\left((M, 0)\left(x_{0} ; y_{0}\right)+p\right)^{\top} \lambda_{1}=0 \\
\left(N x_{0}+Q y_{0}+q\right)^{\top} \lambda_{2}=0 \\
\left(A x_{0}+B y_{0}+b\right)^{\top} \lambda_{3}=0 .
\end{gathered}
$$

From (3.6), we can easily deduce that

$$
\begin{aligned}
\omega^{\top}\left(x_{0} ; y_{0}\right)= & \left\{(M, 0)^{\top} \lambda_{1}+(N, Q)^{\top} \lambda_{2}+(A, B)^{\top} \lambda_{3}+(C, D)^{\top} \lambda_{4}\right\}^{\top}\left(x_{0} ; y_{0}\right) \\
= & \lambda_{1}^{\top}(M, 0)\left(x_{0} ; y_{0}\right)+\lambda_{2}^{\top}(N, Q)\left(x_{0} ; y_{0}\right) \\
& +\lambda_{3}^{\top}(A, B)\left(x_{0} ; y_{0}\right)+\lambda_{4}^{\top}(C, D)\left(x_{0} ; y_{0}\right) \\
= & -\lambda_{1}^{\top} p-\lambda_{2}^{\top} q-\lambda_{3}^{\top} b-\lambda_{4}^{\top} d .
\end{aligned}
$$

Thus, for any $(x ; y) \in R^{2 n}$, from the first equation in (3.6), we have 


$$
\begin{aligned}
{\left[\omega^{\top}\left((x ; y)-\left(x_{0} ; y_{0}\right)\right)\right]_{-}=} & \left\{\lambda_{1}^{\top}((M, 0)(x ; y)+p)+\lambda_{2}^{\top}((N, Q)(x ; y)+q)\right. \\
& \left.+\lambda_{3}^{\top}((A, B)(x ; y)+b)+\lambda_{4}^{\top}((C, D)(x ; y)+d)\right\}_{-} \\
\leq & \left\{\lambda_{1}^{\top}((M, 0)(x ; y)+p)\right\}_{-}+\left\{\lambda_{2}^{\top}((N, Q)(x ; y)+q)\right\}_{-} \\
& +\left\{\lambda_{3}^{\top}((A, B)(x ; y)+b)\right\}_{-}+\left\{\lambda_{4}^{\top}((C, D)(x ; y)+d)\right\}_{-} \\
\leq & \lambda_{1}^{\top}\{(M, 0)(x ; y)+p\}_{-}+\lambda_{2}^{\top}\{(N, Q)(x ; y)+q\}_{-} \\
& +\lambda_{3}^{\top}\{(A, B)(x ; y)+b\}_{-} \\
& +\left\{\lambda_{4}\right\}_{-}^{\top}\{(C, D)(x ; y)+d\}_{+}+\left\{\lambda_{4}\right\}_{+}^{\top}\{(C, D)(x ; y)+d\}_{-} \\
\leq & \left\|\lambda_{1}\right\|\left\|\{(M, 0)(x ; y)+p\}_{-}\right\|+\left\|\lambda_{2}\right\|\left\|\{(N, Q)(x ; y)+q\}_{-}\right\| \\
& +\left\|\lambda_{3}\right\|\left\|\{(A, B)(x ; y)+b\}_{-}\right\|+v\|(C, D)(x ; y)+d\|,
\end{aligned}
$$

Where $v \geq 0$ is a constant. Let $\tau=\max \left\{\left\|\lambda_{1}\right\|,\left\|\lambda_{2}\right\|,\left\|\lambda_{3}\right\|, v\right\}$, then the desired result follows.

Now, we are at the position to state our results.

Theorem 3.3. Suppose that Assumption 2.1 holds. Then, there exists a constant $\eta>0$ such that for any $(x ; y) \in R^{2 n}$, there exists $\left(x^{*} ; y^{*}\right) \in X^{*}$ such that

$$
\left\|(x ; y)-\left(x^{*} ; y^{*}\right)\right\| \leq \eta\left\{s(x, y)+s(x, y)^{1 / 2}\right\}
$$

where

$$
\begin{aligned}
s(x, y)= & \left\|(M x+p)_{-}\right\|+\left\|(N x+Q y+q)_{-}\right\| \\
& +\left\|(A x+B y+b)_{-}\right\|+\|C x+D y+d\|+\left[(M x+p)^{\top}(N x+Q y+q)\right]_{+} .
\end{aligned}
$$

Proof. Using Corollary 2.3 and Lemma 3.1, there exists a constant $\mu_{1}>0$, for any $(x ; y) \in R^{2 n}$, and there exists $\left(x^{*} ; y^{*}\right) \in X^{*}$ such that

$$
\begin{aligned}
\left\|(x ; y)-\left(x^{*} ; y^{*}\right)\right\| \leq \mu_{1}\{ & \left\|(M x+p)_{-}\right\|+\left\|(N x+Q y+q)_{-}\right\| \\
+ & \left\|(A x+B y+b)_{-}\right\|+\|C x+D y+d\|
\end{aligned}
$$


Journal of Applied Mathematics

$$
\begin{aligned}
& +\|\left[\left(\left((M, 0)^{\top}(N, Q)+(N, Q)^{\top}(M, 0)\right)\left(x_{0} ; y_{0}\right)\right.\right. \\
& \left.\left.+(M, 0)^{\top} q+(N, Q)^{\top} p\right)^{\top}\left((x ; y)-\left(x_{0} ; y_{0}\right)\right)\right]_{+} \| \\
& \left.+\left\|\left((M, 0)^{\top}(N, Q)+(N, Q)^{\top}(M, 0)\right)\left((x ; y)-\left(x_{0} ; y_{0}\right)\right)\right\|\right\},
\end{aligned}
$$

Where $\left(x_{0} ; y_{0}\right)$ is a solution of EMLCP. Now, we consider the right-hand-side of expression (3.11).

Firstly, by Assumption 2.1, we obtain that

$$
H(x, y)=(M x+p)^{\top}(N x+Q y+q)
$$

is a convex function. For any $(x ; y) \in R^{2 n}$, we have

$$
\begin{aligned}
& H(x, y)-H\left(x_{0} ; y_{0}\right) \geq[\left((M, 0)^{\top}(N, Q)+(N, Q)^{\top}(M, 0)\right)\left(x_{0} ; y_{0}\right) \\
&\left.+(M, 0)^{\top} q+(N, Q)^{\top} p\right]^{\top}\left((x ; y)-\left(x_{0} ; y_{0}\right)\right) .
\end{aligned}
$$

Combining this with $H\left(x_{0} ; y_{0}\right)=0$, we can deduce that

$$
\begin{aligned}
& \left\{\left[\left((M, 0)^{\top}(N, Q)+(N, Q)^{\top}(M, 0)\right)\left(x_{0} ; y_{0}\right)+(M, 0)^{\top} q+(N, Q)^{\top} p\right]^{\top}\left((x ; y)-\left(x_{0} ; y_{0}\right)\right)\right\}_{+} \\
& \quad \leq\left[(M x+p)^{\top}(N x+Q y+q)\right]_{+} .
\end{aligned}
$$

Secondly, we consider the last item in (3.11). By Assumption 2.1, there exists a constant $\mu_{2}>0$ such that for any $(x ; y) \in R^{2 n}$,

$$
\begin{aligned}
& \left\|\left((M, 0)^{\top}(N, Q)+(N, Q)^{\top}(M, 0)\right)\left((x ; y)-\left(x_{0} ; y_{0}\right)\right)\right\|^{2} \\
& \quad \leq \mu_{2}\left((x ; y)-\left(x_{0} ; y_{0}\right)\right)^{\top}\left((M, 0)^{\top}(N, Q)+(N, Q)^{\top}(M, 0)\right)\left((x ; y)-\left(x_{0} ; y_{0}\right)\right) \\
& \quad=2 \mu_{2}\left\{(M x+p)^{\top}(N x+Q y+q)-\left(M x_{0}+p\right)^{\top}\left(N x_{0}+Q y_{0}+q\right)\right.
\end{aligned}
$$




$$
\begin{gathered}
-\left[\left((M, 0)^{\top}(N, Q)+(N, Q)^{\top}(M, 0)\right)\left(x_{0} ; y_{0}\right)+(M, 0)^{\top} q+(N, Q)^{\top} p\right]^{\top} \\
\left.\times\left((x ; y)-\left(x_{0} ; y_{0}\right)\right)\right\} \\
\leq \mu_{2}\left[(M x+p)^{\top}(N x+Q y+q)\right]_{+}+2 \mu_{2}\left\{\left[\left((M, 0)^{\top}(N, Q)+(N, Q)^{\top}(M, 0)\right)\left(x_{0} ; y_{0}\right)\right.\right. \\
\left.\left.+(M, 0)^{\top} q+(N, Q)^{\top} p\right]^{\top}\left((x ; y)-\left(x_{0} ; y_{0}\right)\right)\right\}_{-} \\
\leq 2 \mu_{2}\left[(M x+p)^{\top}(N x+Q y+q)\right]_{+}+2 \mu_{2} \tau\left(\left\|(M x+p)_{-}\right\|+\left\|(N x+Q y+q)_{-}\right\|\right. \\
\left.+\left\|(A x+B y+b)_{-}\right\|+\|C x+D y+d\|\right),
\end{gathered}
$$

where the first equality is based on the Taylor expansion of function $H(x, y)$ on $\left(x_{0} ; y_{0}\right)$ point, the second inequality follows from the fact that $\left(x_{0} ; y_{0}\right)$ is a solution of EMLCP and the fact that $a+b \leq a_{+}+b_{+}$for any $a, b \in R$, and the last inequality is based on Lemma 3.2. By (3.11)-(3.15), we have that (3.9) holds.

The error bound obtained in Theorem 3.3 coincides with that of Theorem 2.4 in [11] for the linear complementarity problem, and it is also an extension of Theorem 2.7 in [7] and Corollary 2 in [14].

Theorem 3.4. Suppose that the assumption of Theorem 2.5 holds. Then, there exists a constant $\eta_{1}>0$, such that for any $(x ; y) \in R^{2 n}$, there exists a solution $\left(x^{*} ; y^{*}\right) \in X^{*}$ such that

$$
\left\|(x ; y)-\left(x^{*} ; y^{*}\right)\right\| \leq \eta_{1} s(x, y)
$$

where $s(x, y)$ is defined in Theorem 3.3.

Proof. From Theorem 2.5, using the proof technique is similar to that of Theorem 3.3. For any $(x ; y) \in R^{2 n}$, there exist $\left(x^{*} ; y^{*}\right) \in X^{*}$ and a constant $\mu_{4}>0$ such that

$$
\begin{aligned}
\left\|(x ; y)-\left(x^{*} ; y^{*}\right)\right\| \leq \mu_{4}\{ & \left\|(M x+p)_{-}\right\|+\left\|(N x+Q y+q)_{-}\right\| \\
+ & \left\|(A x+B y+b)_{-}\right\|+\|C x+D y+d\| \\
+ & \|\left[\left(\left((M, 0)^{\top}(N, Q)+(N, Q)^{\top}(M, 0)\right)\left(x_{0} ; y_{0}\right)\right.\right. \\
& \left.\left.\left.+(M, 0)^{\top} q+(N, Q)^{\top} p\right)^{\top}\left((x ; y)-\left(x_{0} ; y_{0}\right)\right)\right]_{+} \|\right\} .
\end{aligned}
$$

Combining this with (3.14), we can deduce that (3.16) holds. 


\section{Conclusion}

In this paper, we presented the solution Characterization, and also established global error bounds on the extended mixed linear complementarity problems which are the extensions of those for the classical linear complementarity problems. Surely, we may use the error bound estimation to establish quick convergence rate of the noninterior path following method for solving the EMLCP just as was done in [14], and this is a topic for future research.

\section{Acknowledgments}

This work was supported by the Natural Science Foundation of China (Grant no. 11171180,11101303), Specialized Research Fund for the Doctoral Program of Chinese Higher Education (20113705110002), and Shandong Provincial Natural Science Foundation (ZR2010AL005, ZR2011FL017).

\section{References}

[1] R. W. Cottle, J.-S. Pang, and R. E. Stone, The Linear Complementarity Problem, Academic Press, New York, NY, USA, 1992.

[2] F. Facchinei and J. S. Pang, Finite-Dimensional Variational Inequality and Complementarity Problems, Springer, New York, NY, USA, 2003.

[3] M. C. Ferris and J. S. Pang, "Engineering and economic applications of complementarity problems," Society for Industrial and Applied Mathematics, vol. 39, no. 4, pp. 669-713, 1997.

[4] L. Walras, Elements of Pure Economics, George Allen and Unwin, London, UK, 1954.

[5] S. Karamardian, "Generalized complementarity problem," Journal of Optimization Theory and Applications, vol. 8, pp. 161-168, 1971.

[6] G. J. Habetler and A. L. Price, "Existence theory for generalized nonlinear complementarity problems," Journal of Optimization Theory and Applications, vol. 7, pp. 223-239, 1971.

[7] O. L. Mangasarian and T. H. Shiau, "Error bounds for monotone linear complementarity problems," Mathematical Programming, vol. 36, no. 1, pp. 81-89, 1986.

[8] O. L. Mangasarian, "Error bounds for nondegenerate monotone linear complementarity problems," Mathematical Programming, vol. 48, no. 3, pp. 437-445, 1990.

[9] J.-S. Pang, "Error bounds in mathematical programming," Mathematical Programming, vol. 79, no. 1-3, pp. 299-332, 1997.

[10] Z.-Q. Luo, O. L. Mangasarian, J. Ren, and M. V. Solodov, “New error bounds for the linear complementarity problem," Mathematics of Operations Research, vol. 19, no. 4, pp. 880-892, 1994.

[11] O. L. Mangasarian and J. Ren, "New improved error bounds for the linear complementarity problem," Mathematical Programming, vol. 66, no. 2, pp. 241-255, 1994.

[12] R. Mathias and J.-S. Pang, "Error bounds for the linear complementarity problem with a P-matrix," Linear Algebra and its Applications, vol. 132, pp. 123-136, 1990.

[13] A. J. Hoffman, "On approximate solutions of systems of linear inequalities," Journal of Research of the National Bureau of Standards, vol. 49, pp. 263-265, 1952.

[14] J. Zhang and N. Xiu, "Global s-type error bound for the extended linear complementarity problem and applications," Mathematical Programming B, vol. 88, no. 2, pp. 391-410, 2000. 


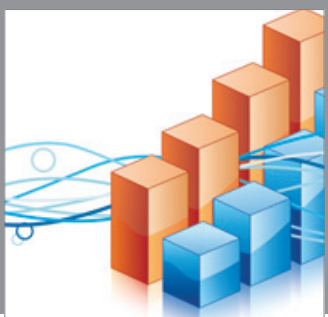

Advances in

Operations Research

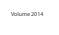

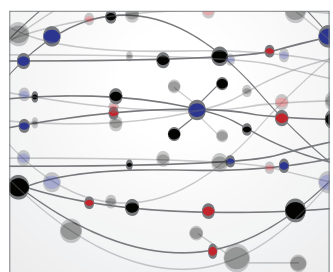

\section{The Scientific} World Journal
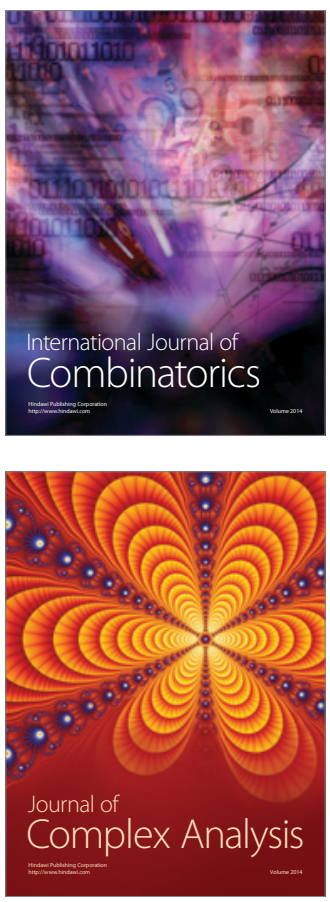

International Journal of

Mathematics and

Mathematical

Sciences
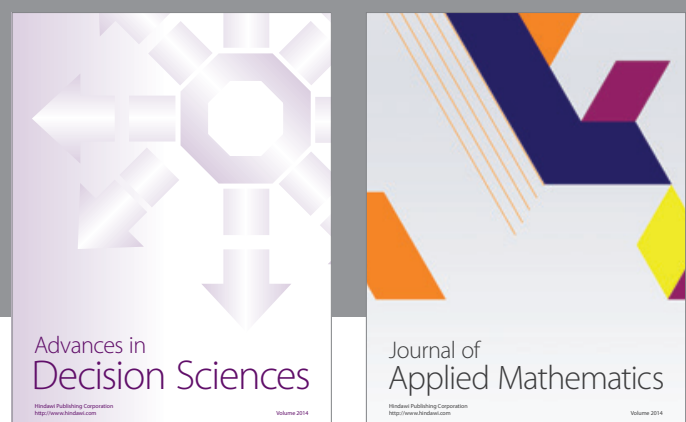

Journal of

Applied Mathematics
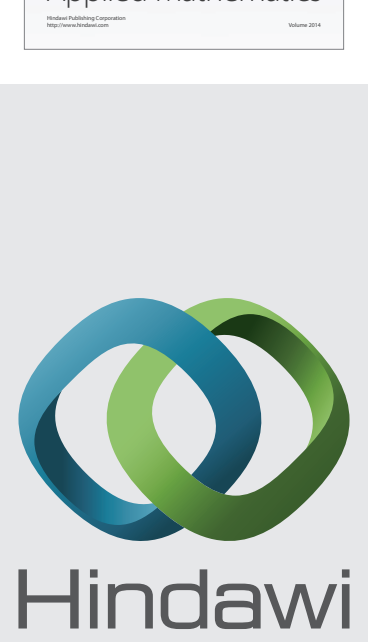

Submit your manuscripts at http://www.hindawi.com
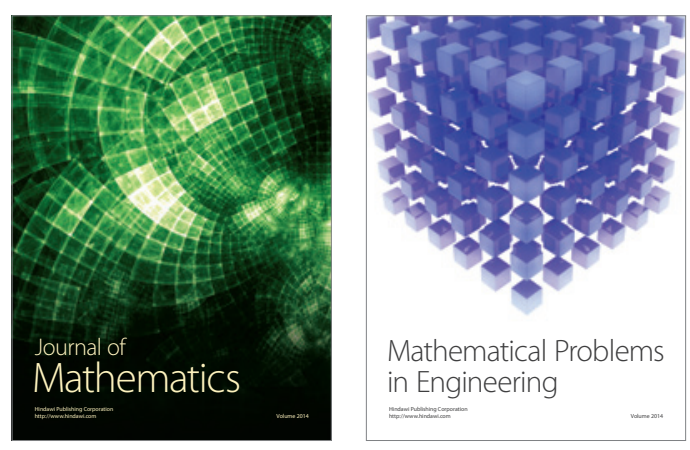

Mathematical Problems in Engineering
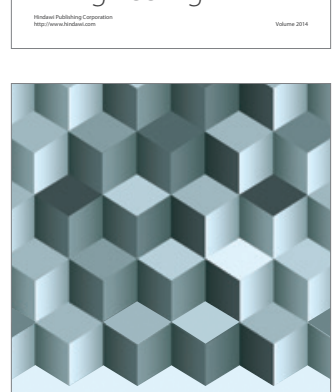

Journal of

Function Spaces
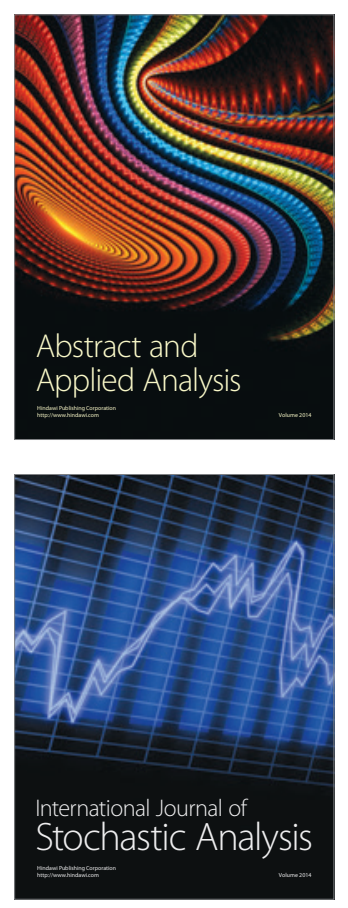

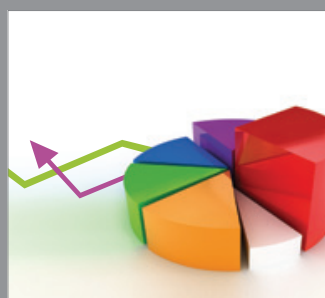

ournal of

Probability and Statistics

Promensencen
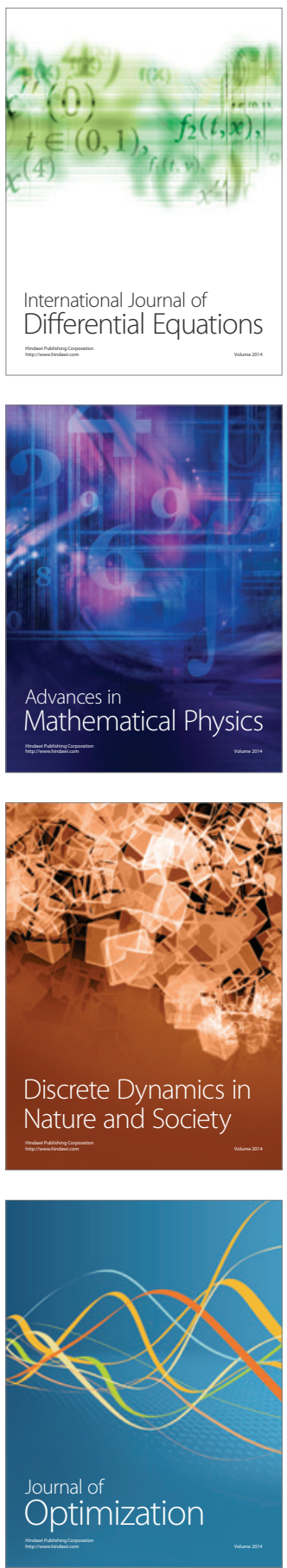